\title{
Near-Infrared Transit Photometry of Extra-Solar Planet HAT-P-54b
}

\author{
Haruka Tabata, Yoichi Itoh \\ Nishi-Harima Astronomical Observatory, Center for Astronomy, University of Hyogo, Hyogo, Japan \\ Email: yitoh@nhao.jp
}

How to cite this paper: Tabata, H. and Itoh, Y. (2020) Near-Infrared Transit Photometry of Extra-Solar Planet HAT-P-54b. International Journal of Astronomy and Astrophysics, 10, 89-96.

https://doi.org/10.4236/ijaa.2020.102007

Received: April 3, 2020

Accepted: May 8, 2020

Published: May 11, 2020

Copyright $\odot 2020$ by author(s) and Scientific Research Publishing Inc. This work is licensed under the Creative Commons Attribution International License (CC BY 4.0).

http://creativecommons.org/licenses/by/4.0/

\begin{abstract}
The results of near-infrared photometric observations of a transit event of an extrasolar planet HAT-P-54b are presented herein. Precise near-infrared photometry was carried out using the Nayuta 2 m telescope at Nishi-Harima Astronomical Observatory, Japan and Nishi-Harima Infrared Camera (NIC). $170 \mathrm{~J}-\mathrm{H}$-, and $\mathrm{Ks}$-band images were taken in each band in 196 minutes. The flux of the planetary system was observed to decrease during the transit event. While the $K s$-band transit depth is similar to that in the $r$-band, the $J$ - and $H$-band transits are deeper than those in the Ks-band. We constructed simple models of the planetary atmosphere and found that the observed transit depths are well reproduced by inflated atmosphere containing $\mathrm{H}_{2} \mathrm{~S}$ molecule.
\end{abstract}

\section{Keywords}

Planetary Systems, Infrared: Planetary Systems

\section{Introduction}

Astronomical observations of extrasolar planets have revealed a rich diversity: for instance, planets ranging from Earth-sized to giant Jupiter-sized ones; besides, there are Jupiter-mass planets orbiting very close to the host star, while there are others with an eccentric orbit. Furthermore, the recent transit observations have revealed diversity not only on the planetary bodies and orbits, but also in terms of the planetary atmosphere: a fraction of the hot Jupiters have an inflating atmosphere, while for others, the atmosphere seems to be escaping from the planet. Detailed infrared observations of the transit events of the extrasolar planets can be used to reveal the thermal structure and temperature inversion of their atmosphere.

Photometric and spectroscopic observations of a transit event reveal the chemi- 
cal composition of the atmosphere of an extrasolar planet. Transit depth is deep at the wavelength where the atoms or molecules in the atmosphere absorb the light of the host star. To date, the presence of sodium, potassium [1], and $\mathrm{H}_{2} \mathrm{O}$ [2] has been confirmed in the atmosphere of the extrasolar planets, while molecules such as $\mathrm{CO}, \mathrm{CO}_{2}$, and $\mathrm{CH}_{4}$ have also been detected. Furthermore, solid particles in the form of clouds and haze have also been identified in the atmospheres of extrasolar planets.

HAT-P-54b is a hot-Jupiter orbiting a low-mass main-sequence star HAT-P-54 with an orbital period of 3.8 days. The mass and radius of the star are 0.645 solar-mass and 0.617 solar-radius, respectively. The transit events of this planetary system were observed in the $r$-band with the HAT-6 instrument at Fred Lawrence Whipple Observatory (FLWO) in Arizona and the HAT-9 instrument at Mauna Kea Observatory in Hawaii, and in the $i$-band with the KeplerCam imager on the FLWO $1.2 \mathrm{~m}$ telescope [3]. This observations yield that the planet-to-star radius ratio is 0.157 , i.e., the radius of the planet is $134,977 \mathrm{~km}$, in the $r$-band. Combined with the Doppler shift measurements, the mass and orbital inclination of the planet were derived to be 0.76 Jupiter mass and $87.04^{\circ}$, respectively, using which, its surface gravity was deduced to be $21.09 \mathrm{~m} \cdot \mathrm{s}^{-2}$.

We conducted near-infrared photometry of the transit event of HAT-P-54 using a unique three-color camera: the transit light curves were simultaneously recorded in three bands. Infrared wavelength observations offer a great advantage for investigating the chemical composition of the atmosphere of an extrasolar planet, because the surface temperature of most such planets is less than $2000 \mathrm{~K}$, so that most species form molecules with vibration bands in the infrared wavelengths.

\section{Observations and Data Reduction}

The near-infrared transit observations of HAT-P-54 were carried out on March 19, 2015 using the Nishi-Harima Infrared Camera (NIC) mounted on the Nayuta $2 \mathrm{~m}$ telescope at Nishi-Harima Astronomical Observatory, Japan. NIC has three $1024 \times 1024 \mathrm{HgCdTe}$ arrays with a field of view of $2.73^{\prime} \times 2.73^{\prime}$. The images in the $J$-, $H_{-}$, and $K s$-bands were taken simultaneously. Near-infrared magnitudes of HAT-P-54 are $11.15 \mathrm{mag}, 10.49 \mathrm{mag}$, and $10.33 \mathrm{mag}$ in the $J$-, $H$-, and $K s$-bands, respectively. The transit event was predicted to begin at 21:48 and end at 23:36 (JST). Therefore, we observed the planetary system between 21:08 and 24:24 (JST). As a reference star, 2MASS J06393662 +2530129 , which is a main-sequence star located 1.37' away from HAT-P-54, was also observed simultaneously. Its near-infrared magnitudes are $10.95 \mathrm{mag}, 10.37 \mathrm{mag}$, and $10.20 \mathrm{mag}$ in the $J-, H^{-}$, and $K s$-bands, respectively. Ten frames of $30 \mathrm{~s}$ exposure each were taken with $10 "$ radius telescope dithering. In total, 170 frames were obtained in each $J^{-}, H^{-}$, and $K s$-band. The seeing condition was 3.0", 2.7", and $2.4^{\prime \prime}$ in the $J-, H_{-}$, and $K s$-bands, respectively.

The data were processed using the Image Reduction and Analysis Facility (IRAF). The object frames were calibrated in the standard manner, namely by 
dark subtraction and flat fielding with the twilight frames. Next, we created sky frames by calculating the median of the dithered 10 frames. Each sky frame was then subtracted from the flat-fielded object frame. A vertical stripe pattern remained in the sky-subtracted image. We estimated the intensity of the stripe pattern in the region without objects, and removed this count from the sky-subtracted image. The fluxes of the target and reference stars were derived by aperture photometry: the aperture radius was set to the FWHM of the PSF, so that the standard deviation of the relative flux, as shall be explained later, shows minimum at the time out of the transit event. The flux of the target star was divided by that of the reference star; we call this the relative flux. The relative fluxes before and after the transit should be the same, unless the target or reference star is a variable star. However, the average of 32 relative fluxes before the transit was larger than that of 41 relative fluxes after the transit. We fitted these 73 fluxes a linear function, and all relative fluxes were divided by the function, so that the average of the out-of-transit relative flux is unity. Nevertheless, the relative flux still has an artificial pattern. The position of the target and reference stars in the image were changed by telescope dithering. We noticed large relative flux when the target star was imaged in the lower part of the image, which could be due to the imperfection of the flat fielding. We calculated the average of the normalized relative fluxes out of the transit for each dithering position, and then subtracted the average from each normalized relative flux. Finally, we added unity.

\section{Results}

The transit event of HAT-P-54b was detected in three bands in the near-infrared wavelengths (Figure 1). We fitted the light curves to the relative flux at the EXOFAST website (http://astroutils.astronomy.ohio-state.edu/exofast/exofast.shtml, [4]), and then derived the transit depths. The values listed in Table 1 were used as the input physical and orbital parameters for the host star and planet, respectively. The transit depths are derived to be $0.0273 \pm 0.0010,0.0282 \pm 0.0007$, and $0.0246 \pm 0.0010$ in the $J-, H$-, and $K s$-bands, respectively. The transit depths in the $J$ - and $H$-bands are deeper than those in the $K s$-band. The EXOFAST website also provides the radius ratio of an extrasolar planet and its host star; the derived radius ratios are larger in the $J$ - and $H$-bands than in the $K s$-band (Table 2). Furthermore, we fitted a light curve to the $i$-band transit data obtained using KeplerCam on the FLWO $1.2 \mathrm{~m}$ telescope at JD 2456310 [3].

\section{Discussion}

The transit depths are different in different bands. The depth in the $K s$-band is similar to the depth in the $r$-band [3]. On the other hand, the depths in the $J$ and $H$-bands are deeper than those in the $r$-and $K s$-bands (Figure 2).

The transit depth does not change significantly with wavelengths, if the planetary atmosphere is cloudy or hazy. However, if the planetary atmosphere is clear, the depth changes with the wavelength. This is not only due to the absorption of 
photons from the host star by the atoms and molecules in the atmosphere of the planet, but also the Rayleigh scattering of the atoms and molecules at short wavelengths. However, we cannot discuss the Rayleigh scattering for HAT-P-54b, owing to the lack of transit observations at short wavelengths.

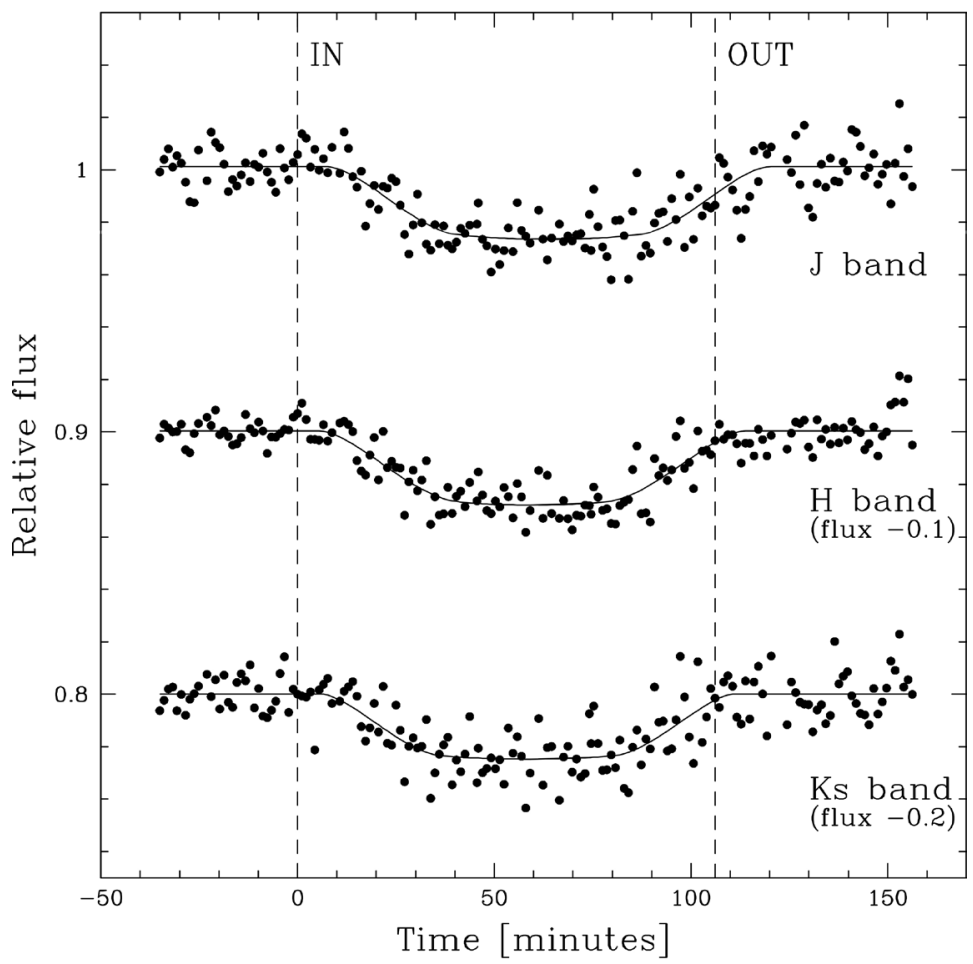

Figure 1. Near-infrared relative fluxes of the transit event of HAT-P-54b. The fluxes of HAT-P-54 were normalized by those of the reference star. Solid lines represent the light curves fitted with the differential evolution Markov chain Monte Carlo method.

Table 1. Physical parameters of the host star and the orbital parameters of the planet.

\begin{tabular}{cc}
\hline Parameter & Value \\
\hline Effective temperature & $4390 \pm 50 \mathrm{~K}$ \\
Metallicity & $-0.127 \pm 0.08$ \\
Surface gravity & $4.467 \pm 0.012\left[\log \mathrm{cm} \cdot \mathrm{s}^{-2}\right]$ \\
Orbital period of the planet & $3.799847 \pm 1.4 \times 10^{-5} \mathrm{day}$ \\
Orbital inclination of the planet & $87.040 \pm 0.084 \mathrm{degree}$ \\
\hline
\end{tabular}

Table 2. Radius ratios of the planet to the host star.

\begin{tabular}{cl}
\hline Band & Radius ratio \\
\hline$i$ & $0.162 \pm 0.001$ \\
$J$ & $0.165 \pm 0.003$ \\
$H$ & $0.168 \pm 0.002$ \\
$K S$ & $0.157 \pm 0.003$ \\
\hline
\end{tabular}




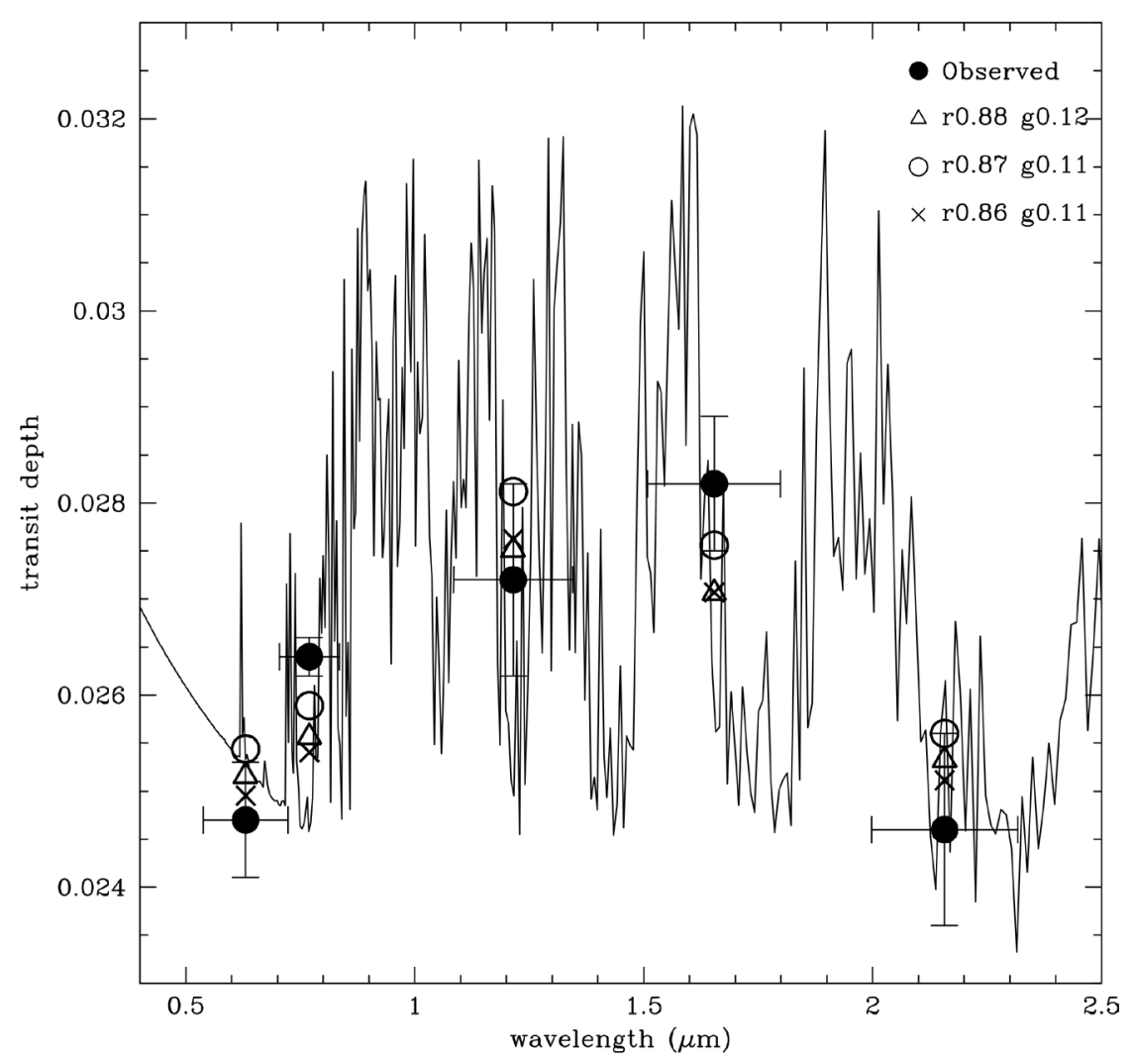

Figure 2. The transit depths as a function of wavelengths. The transit depth is the decrease of the relative flux due to the planet's transit across the host star, normalized by the relative flux out of the transit event. Filled circles are the observed depths and the solid line shows the transit depth spectrum of the model atmosphere. Open circles indicate the transit depths of the best-fit model atmosphere in optical and near-infrared broad-bands, where the radius and surface gravity are $87 \%$ and $11 \%$ of the original values, respectively.

We constructed the transmission model spectra of the planet using the Planetary Spectrum Generator [5] (https://psg.gsfc.nasa.gov/), which calculates the transit depths as a function of the wavelengths. The temperature of the host star was set to $4390 \mathrm{~K}$, its radius to 0.62 solar-radius, and the distance between the star and the planet to $0.0409 \mathrm{AU}$. For the planetary atmosphere, we used a pressure-temperature profile of a giant planet [6]; the equilibrium temperature was $818 \mathrm{~K}$, and no scattering aerosols were considered. The free parameters were the radius of the planet, the scale height of the atmosphere, and a species of an atom or molecule. We varied the scale height by changing the value of the surface gravity. We constructed atmospheric models by varying the radius and surface gravity of the planet in steps of $1 \%$ of the original value, respectively. The transit depths of the HAT-P-54 system have thus far been deduced only at five points by the photometric observations. To simplify the model, only one atom or molecule species was included in the model atmosphere. Sixty species of atoms or molecules were investigated. We used the EXO-Transmit database [7] for the cross section when available, and the HITRAN database otherwise. Among the constructed transmission spectra, the spectra of the atmosphere containing $\mathrm{OH}$, 
$\mathrm{HCl}$, and $\mathrm{H}_{2} \mathrm{~S}$ showed deep absorptions in the $J$ - and $H$-bands. We multiplied the transmission curves of the $r^{-}, i_{-}, J-, H$-, and $K s$-band filters to the transmission spectra and then calculated the transit depth in each band.

The best fit spectrum was the spectrum of the atmosphere containing $\mathrm{H}_{2} \mathrm{~S}$ molecules (Figure 2): namely, the planetary radius of $117,430 \mathrm{~km}$, which is $87 \%$ of the radius estimated from the $r^{\prime}$-band transit data [3], and surface gravity of 2.32 $\mathrm{m} \cdot \mathrm{s}^{-2}$, which is $11 \%$ of the surface gravity calculated from the planetary radius derived from the $r$-band transit data and the planetary mass derived from the Doppler shift measurement [3] (Table 3). The scale height of the atmosphere was about 10 times larger than that in equilibrium, which indicates that the atmosphere of HAT-P-54b is inflating.

The sulfur chemistry in the atmosphere of hot Jupiters investigated by several studies indicates that the HS and $\mathrm{S}_{2}$, which are photochemically and thermochemically generated from $\mathrm{H}_{2} \mathrm{~S}$, absorb substantial amounts of light at high altitudes, causing stratospheric temperature inversions [8]: $\mathrm{H}_{2} \mathrm{~S}$ absorbs UV photons of wavelengths between 200 and $260 \mathrm{~nm}$, and then dissociates into mercapto (HS) and $\mathrm{H}$. We estimated the UV-C flux $(\lambda=121.6-280 \mathrm{~nm})$ at the surface of HAT-P-54b. The spectral type of the host star of the planet, i.e., HAT-P-54 is late-K. The $\mathrm{UV}-\mathrm{C}$ flux of the Sun at $1 \mathrm{AU}$ is $3.38 \mathrm{Wm}^{-2}$ and that of a late-K dwarf at $1 \mathrm{AU}$ is $0.506 \mathrm{Wm}^{-2}$ [9]. The semi-major axis of the orbit of HAT-P-54b is $0.0412 \mathrm{AU}$. We calculated the UV-C flux at the surface of the planet as

$$
0.506 \times\left(\frac{1}{0.0412}\right)^{2}=298 \mathrm{Wm}^{-2},
$$

which is 88 times stronger than the flux at the surface of the Earth.

It was reported that while $\mathrm{H}_{2} \mathrm{~S}$ is abundant in the lower atmosphere $(P>0.001$ bar), $\mathrm{CO}$ is more abundant than $\mathrm{H}_{2} \mathrm{~S}$ at any altitude [8], as it exhibits strong absorption bands in the $K s$-band. However, our observations showed that the transit depth in the $K s$-band is as shallow as that in the $r$-band; thus, we did not find any evidence of $\mathrm{CO}$ absorption.

The observation possibility of $\mathrm{H}_{2} \mathrm{~S}$ in hot Jupiters using the JWST telescope is examined [10]. It is indicated that $\mathrm{H}_{2} \mathrm{~S}$ is the dominant species at pressure levels between $1 \times 10^{-4}$ and $1 \times 10^{4}$ bar in such atmospheres. The infrared model spectra of hot Jupiters observed by JWST were constructed in the wavelength range from 2.0 to $11 \mu \mathrm{m}$ : The absorption features of $\mathrm{H}_{2} \mathrm{~S}$ between 2.6 and $2.8 \mu \mathrm{m}$ and between 3.5 and $4.1 \mu \mathrm{m}$ can be detected in the transmission spectra for the planet with an equilibrium temperature higher than $1500 \mathrm{~K}$.

Table 3. Physical parameters of the planet.

\begin{tabular}{cc}
\hline parameter & value \\
\hline Equilibrium temperature & $818 \mathrm{~K}$ \\
Radius & $117,430 \mathrm{~km}$ \\
Surface gravity & $2.32 \mathrm{~m} \cdot \mathrm{s}^{-2}$ \\
\hline
\end{tabular}


We measured the transit depths of HAT-P-54b only in three bands, and despite combining our results with those of a previous study, the transit depths could be determined only in five bands by photometry. Therefore, our conclusion that the atmosphere of HAT-P-54b contains $\mathrm{H}_{2} \mathrm{~S}$ may not be a unique solution. To seek other species and to confirm $\mathrm{H}_{2} \mathrm{~S}$ in the atmosphere of this planet, photometric observations in other wavelengths and spectroscopic observations are required. More complicated atmospheric models including stratospheric thermal inversion and altitude distribution of atoms and molecules are mandatory for the comparison.

\section{Conclusion}

We conducted near-infrared photometry of the transit event of an extrasolar planet, HAT-P-54b. Precise near-infrared photometry was carried out using the Nayuta $2 \mathrm{~m}$ telescope and Nishi-Harima Infrared Camera (NIC). 170 near-infrared images were taken in each $J$-, $H$-, and $K s$-band in 196 minutes. The flux of the planetary system was observed to decrease during the transit event. While the transit depth in the $K s$-band was $0.0246 \pm 0.0010$, which is similar to that in the $r$-band, greater depths were observed in the $J$ - and $H$-bands, i.e., $0.0273 \pm 0.0010$ and $0.0282 \pm 0.0007$, respectively. Furthermore, we constructed simple models of the planetary atmosphere and found that the observed transit depths are well reproduced by the inflated atmosphere containing the $\mathrm{H}_{2} \mathrm{~S}$ molecule.

\section{Acknowledgements}

This work was supported by JSPS KAKENHI Grant Number JP17K05390.

\section{Conflicts of Interest}

The authors declare no conflicts of interest regarding the publication of this paper.

\section{References}

[1] Charbonneau, D., Brown, T.M., Noyes, R.W. and Gilliland, R.L. (2002) Detection of an Extrasolar Planet Atmosphere. The Astrophysical Journal, 568, 377-384. https://doi.org/10.1086/338770

[2] Deming, D., Wilkins, A., McCullough, P., et al. (2013) Infrared Transmission Spectroscopy of the Exoplanets HD 209458b and XO-1b Using the Wide Field Camera-3 on the Hubble Space Telescope. The Astrophysical Journal, 774, Article ID: 95. https://doi.org/10.1088/0004-637X/774/2/95

[3] Bakos, G.A., Hartman, J.D., Bhatti, W., et al. (2015) HAT-P-54b: A Hot Jupiter Transiting a $0.64 \mathrm{M}_{\mathrm{e}}$ Star in Field 0 of the K2 Mission. The Astronomical Journal, 149, Article ID: 149. https://doi.org/10.1088/0004-6256/149/4/149

[4] Eastman, J., Gaudi, B.S. and Agol, E. (2013) EXOFAST: A Fast Exoplanetary Fitting Suite in IDL. Publications of the Astronomical Society of the Pacific, 125, 83-112. https://doi.org/10.1086/669497

[5] Villanueva, G.L., Smith, M.D., Protopapa, S., et al. (2018) The Planetary Spectrum Generator (PSG): An Online Simulator of Exoplanets. LPI Contribution, 2065, Article ID: 2017. 
[6] Parmentier, V. and Guillot, T. (2014) A Non-Grey Analytical Model for Irradiated Atmospheres. I. Derivation. Astronomy \& Astrophysics, 562, Article ID: A133. https://doi.org/10.1051/0004-6361/201322342

[7] Kempton, E.M.-R., Lupu, R., Owusu-Asare, A., et al. (2017) Exo-Transmit: An Open-Source Code for Calculating Transmission Spectra for Exoplanet Atmospheres of Varied Composition. Publications of the Astronomical Society of the Pacific, 129, Article ID: 044402. https://doi.org/10.1088/1538-3873/aa61ef

[8] Zahnle, K., Marley, M.S., Freedman R.S., et al. (2009) Atmospheric Sulfur Photochemistry on Hot Jupiters. The Astrophysical Journal Letters, 701, L20-L24. https://doi.org/10.1088/0004-637X/701/1/L20

[9] Rugheimer, S., Segura, A., Kaltenegger, L., et al. (2015) UV Surface Environment of Earth-like Planets Orbiting FGKM Stars through Geological Evolution. The Astrophysical Journal, 806, Article ID: 137. https://doi.org/10.1088/0004-637X/806/1/137

[10] Wang, D., Miguel, Y. and Lunine, J. (2017) Modeling Synthetic Spectra for Transiting Extrasolar Giant Planets: Detectability of $\mathrm{H}_{2} \mathrm{~S}$ and $\mathrm{PH}_{3}$ with the James Web Space Telescope. The Astrophysical Journal, 850, Article ID: 199. https://doi.org/10.3847/1538-4357/aa978e 